\title{
Experimental verification of metamaterial based subwavelength microwave absorbers
}

Kamil Boratay Alici, Filiberto Bilotti, Lucio Vegni, and Ekmel Ozbay

Citation: Journal of Applied Physics 108, 083113 (2010);

View online: https://doi.org/10.1063/1.3493736

View Table of Contents: http://aip.scitation.org/toc/jap/108/8

Published by the American Institute of Physics

\section{Articles you may be interested in}

Ultra-broadband microwave metamaterial absorber

Applied Physics Letters 100, 103506 (2012); 10.1063/1.3692178

A broadband low-reflection metamaterial absorber

Journal of Applied Physics 108, 064913 (2010); 10.1063/1.3485808

Ultrathin multiband gigahertz metamaterial absorbers

Journal of Applied Physics 110, 014909 (2011); 10.1063/1.3608246

High performance optical absorber based on a plasmonic metamaterial

Applied Physics Letters 96, 251104 (2010); 10.1063/1.3442904

Dual band terahertz metamaterial absorber: Design, fabrication, and characterization

Applied Physics Letters 95, 241111 (2009); 10.1063/1.3276072

A novel ultrathin and broadband microwave metamaterial absorber

Journal of Applied Physics 116, 094504 (2014); 10.1063/1.4894824

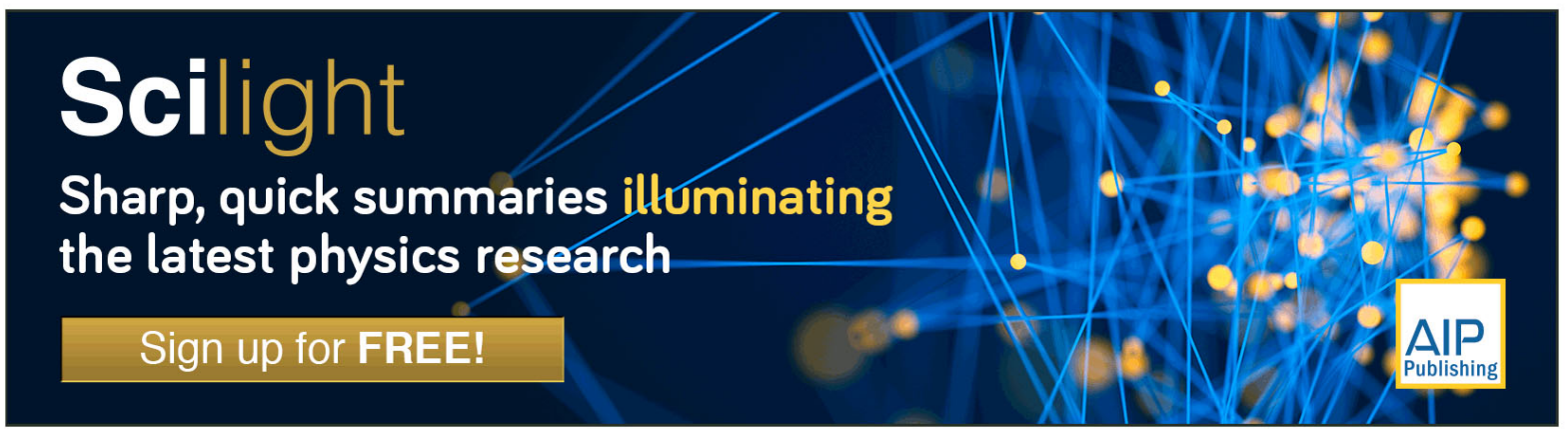




\title{
Experimental verification of metamaterial based subwavelength microwave absorbers
}

\author{
Kamil Boratay Alici, ${ }^{1, a)}$ Filiberto Bilotti, ${ }^{2}$ Lucio Vegni, ${ }^{2}$ and Ekmel Ozbay ${ }^{1,3}$ \\ ${ }^{1}$ Nanotechnology Research Center, Bilkent University, Bilkent, 06800 Ankara, Turkey \\ and Department of Physics, Bilkent University, Bilkent, 06800 Ankara, Turkey \\ ${ }^{2}$ Department of Applied Electronics, University "Roma Tre", Via della Vasca Navale, 84-00146 Rome, Italy \\ ${ }^{3}$ Department of Electrical and Electronics Engineering, Bilkent University, Bilkent, 06800 Ankara, \\ Turkey
}

(Received 5 March 2010; accepted 26 August 2010; published online 29 October 2010)

\begin{abstract}
We designed, implemented, and experimentally characterized electrically thin microwave absorbers by using the metamaterial concept. The absorbers consist of (i) a metal back plate and an artificial magnetic material layer; (ii) metamaterial back plate and a resistive sheet layer. We investigated absorber performance in terms of absorbance, fractional bandwidth, and electrical thickness, all of which depend on the dimensions of the metamaterial unit cell and the distance between the back plate and metamaterial layer. As a proof of concept, we demonstrated a $\lambda / 4.7$ thick absorber of type I, with a $99.8 \%$ absorption peak along with a $8 \%$ fractional bandwidth. We have shown that as the electrical size of the metamaterial unit cell decreases, the absorber electrical thickness can further be reduced. We investigated this concept by using two different magnetic metamaterial inclusions: the split-ring resonator (SRR) and multiple SSR (MSRR). We have also demonstrated experimentally a $\lambda / 4.7$ and a $\lambda / 4.2$ thick absorbers of type II, based on SRR and MSRR magnetic metamaterial back plates, respectively. The absorption peak of the SRR layout is $97.4 \%$, while for the MSRR one the absorption peak is $98.4 \%$. The $10 \mathrm{~dB}$ bandwidths were $9.9 \%$ and $9.6 \%$ for the SRR and MSRR cases, respectively. (C) 2010 American Institute of Physics. [doi:10.1063/1.3493736]
\end{abstract}

\section{INTRODUCTION}

An electromagnetic absorber neither reflects nor transmits the incident radiation. Therefore, the power of the impinging wave is mostly absorbed in the absorber materials. The performance of an absorber depends on its thickness and morphology, and also the materials used to fabricate it. The simples absorber layout is referred to as the Salisbury screen. ${ }^{1}$ It consists of a $377 \Omega$ resistive sheet placed a quarter wavelength $\left(\lambda_{0} / 4\right)$ apart from a metal plate at the center operation frequency $\left(f_{0}\right)$. Salisbury proved that at integer multiples of $\lambda_{0} / 4$, there is a reflection dip, implying resonant absorption. Though the frequency bandwidth of the absorber is inherently limited by its resonant electromagnetic behavior, resonant absorbers find several applications in microwave technology, especially in the reduction in resonant peaks of the radar signature of metallic objects and sharp shapes.

Recent advances in artificial electromagnetic materials have made it possible to control the electromagnetic field by means of properly engineered permittivity and permeability functions. ${ }^{2-27}$ Such materials, usually referred to as metamaterials, even provided simultaneously negative permittivity and permeability, and extended the domains of interactions between the electromagnetic field and the matter. ${ }^{2,4,5,9,12-14,18,21,22,28,29}$ Metamaterial concepts, such as magnetic resonance and negative refraction, have led researchers to come up with new types of antennas, ${ }^{11,15,16,30-34}$ lenses, ${ }^{9,13,22,35}$ and absorbers. ${ }^{36-39}$ Recently, ferromagnetic

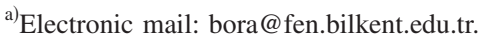

microwire based negative refraction has been demonstrated 40 and related microwave absorbers have attracted attention. ${ }^{41,42}$

The first metamaterial based absorbers have been proposed by Bilotti et al. and Mosallaei and Sarabandi and they are both characterized by an ultrathin absorber thickness and a narrow operation band. ${ }^{37,38,43}$ Later, Padilla's group demonstrated the perfect metamaterial absorber by utilizing the high imaginary part of the metamaterial index of refraction. ${ }^{39}$ In other words, they utilized the lossy nature of metamaterials and achieved a thin absorber with a $\lambda / 2.2$ unit cell dimension. The maximum measured absorption peak was $88 \%$ at $11.5 \mathrm{GHz}$ with a $460 \mathrm{MHz}$ bandwidth $(\Delta f)$. The fractional bandwidth $\left(\Delta f / f_{0}\right)$ was $\mathrm{FBW}=4 \%$, and the metamaterial unit cell was composed of two patterned metallic surfaces separated by a dielectric board: an electric ring resonator on the front and a line strip at the back. One advantage of metamaterial based absorbers is that the operation frequency can be controlled by scaling the constituting unit cells. These techniques that were developed mainly at the microwave frequencies can be applied at higher frequencies until the unit cell dimension and metal skin depth become comparable. ${ }^{44}$

In the present paper, we experimentally verify two metamaterial based configurations made of a planar arrangement of subwavelength inclusions combined with either a metal plate or a resistive sheet. The structure of the paper is as follows. In Sec. II, we present the geometry of the proposed designs. In Sec. III, we describe the experiment setup. In Sec. IV, we present the results of the experimental characterization of the two types of absorbers in terms of resonance mechanism, electrical thickness and inclusion type. 


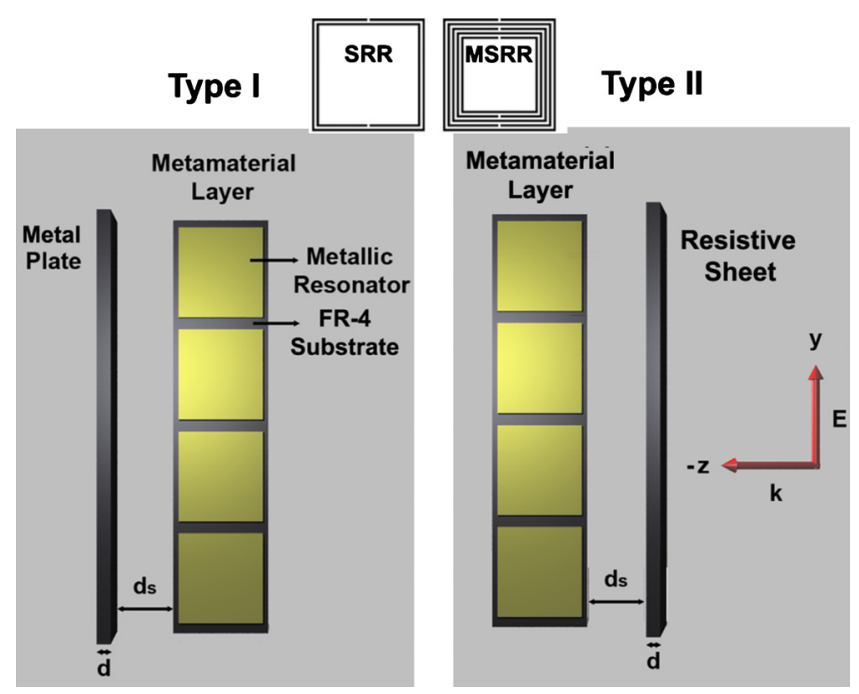

FIG. 1. (Color online) Geometry and schematic of the two absorber designs. Type I absorber consists of an array of magnetic resonators placed in front of a thin aluminum plate. Type II absorber consists of a carbon resistive sheet backed by the same metamaterial layer as for type I. The wavevector (k) of the incident field is in the $z$-direction and the electric field $(\mathbf{E})$ is in the $y$-direction. As metallic resonators we used SRR and MSRR.

\section{DESIGN AND GEOMETRY}

The metamaterial based miniaturized absorbers, which we present in this paper, consist of a back plate made of either metal or a magnetic metamaterial and a front layer made of either a magnetic metamaterial or a resistive sheet, respectively. The sketches of the proposed absorbers are reported in Fig. 1. The metal plate and the resistive sheet thicknesses are $d=0.5 \mathrm{~mm}$ and $d=1.8 \mathrm{~mm}$, respectively. The magnetic metamaterial layer consists of a two-dimensional (2D) array of magnetic inclusions, namely split-ring resonators (SRRs) or multiple SSRs (MSRRs), having a side length of $l=3.2 \mathrm{~mm}$. The parameters of the SRR/MSRR are as follows: strip width $w=0.1 \mathrm{~mm}$, separation between the strips $s=0.1 \mathrm{~mm}$, split (gap) width $g=0.1 \mathrm{~mm}$, number of rings $N=2$ and $N=8$ for the SRR and MSRR, respectively. The substrate on which resonators are etched is FR-4 with thickness $t=1 \mathrm{~mm}$ and a listed relative dielectric constant of $\varepsilon_{r}$ $=4.9(1+i 0.02)$. The thickness of the copper strips in the FR-4 board is $h=18 \mu \mathrm{m}$. In Fig. 1, we show the right view of the absorber configurations and the SRR/MSRR geometry in the inset. The periods in the $x$-direction and $y$-direction are $p_{x}=1.8 \mathrm{~mm}$ and $p_{y}=3.4 \mathrm{~mm}$, respectively. The separation between the back plate and magnetic the metamaterial medium is indicated as $d_{s}$. In the experiments, we used 82 layers in the $x$-direction and 38 unit cells in the $y$-direction, leading to a total transverse dimension of $148 \times 140 \mathrm{~mm}^{2}$.

\section{EXPERIMENTS}

We developed a homemade free-space measurement system that is composed of standard horn antennas, microwave lenses, and a network analyzer operating at around the 3.5-7 $\mathrm{GHz}$ band. All of the elements of the setup were connected by using specifically manufactured aluminum holders, which have been aligned by means of mercury steel bars passing through the corners of the sample holders.

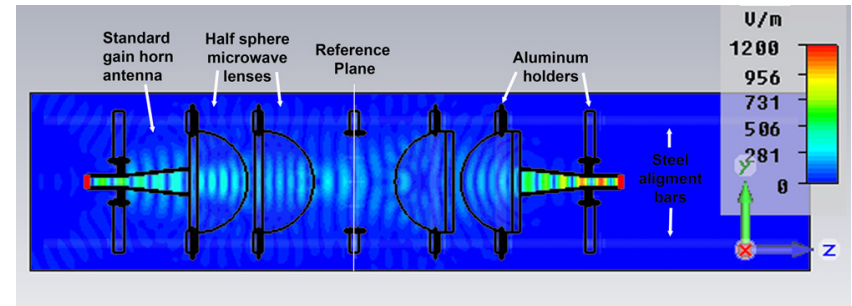

FIG. 2. (Color online) Experimental setup and simulated electric field magnitude distribution at $5 \mathrm{GHz}$. The setup was placed as the steel bars touch the ground and the propagation direction was parallel to the gravitational acceleration. In the simulation the field was propagating in the $z$-direction.

The setup configuration is reported in Fig. 2. The direction of gravity is from right to left, which made the placement of the samples under test easier. We use two hemispherical teflon (the dielectric constant is 2) lenses in front of each of the two $10 \mathrm{~dB}$ gain standard horn antennas. The lenses have been manufactured through the wide spread turning machines and their diameter is $20 \mathrm{~cm}$. We verified the focus distance of the two lenses by direct scanning measurements and compared these data with the simulations. The focus was $f \sim 8 \mathrm{~cm}$ away from the lens and the lateral full width at half maximum of the beam was $6 \mathrm{~cm}$ at around 5 $\mathrm{GHz}$. The simulated magnitude of the electric field at $5 \mathrm{GHz}$, when the reference plane for the sample is left empty is shown in Fig. 2. The simulations have been performed through CST MICROWAVE STUDIO. The diffraction and scattering of the wave from the metallic holders may seem to pose a problem of high noise levels for the transmission-reflection measurements. However, for the limited band 3.5-7 GHz we have seen a quite good calibration response and did not use extra absorbers to reduce the unwanted scattered radiation. On the other hand, it is always possible to further improve the setup by incorporating absorbers at the holders to reduce the scattering effects.

The two horn antennas were connected to a two-port HP8510C vector network analyzer. In a two-port network, the scattering parameters have been measured with a device imbedded between a $50 \Omega$ load and the source. For a complete characterization of a planar sample, we measured the four complex scattering parameters of the two ports: (i) input reflection coefficients with the output port terminated by a matched load $\left(S_{11}\right.$ and $\left.S_{22}\right)$ and (ii) forward transmission (insertion) gain with the output port terminated in a matched load $\left(\mathrm{S}_{21}, \mathrm{~S}_{12}\right)$. Before starting the characterization measurements, we performed a through-reflect-line (TRL) calibration.

For the calibration, we used the same method described by Varadan and co-workers ${ }^{45}$ in 1989. The internal TRL calibration model of the HP 8510C was performed. First, we moved the second antenna and its lenses to a distance of $2 f+\lambda / 4$ from the first one, where $\lambda$ is the wavelength at the midband $(5 \mathrm{GHz})$ and $f$ is the focal distance. We measured the line standard. Second, we moved the second antenna and its two lenses to a position such that the distance between the two lenses was two times the focal length $(2 f)$. Then we measured the through standard. Finally, we placed an aluminum plate at the reference plane that was a focal length away 


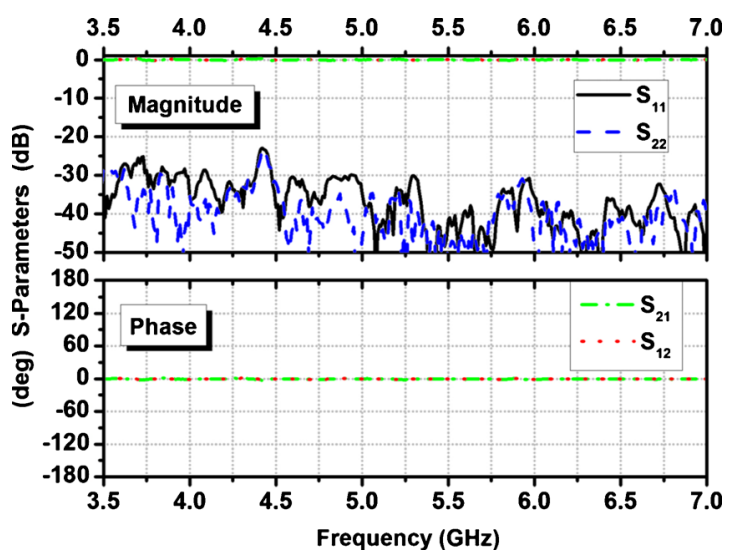

FIG. 3. (Color online) Measured scattering (s) parameters of the free-space after TRL calibration.

from both lenses, and measured the reflect standard. Now that our free-space calibration was ready, we moved on to the test measurements.

In Fig. 3 we show the free-space measurement results in terms of both the transmission magnitude/phase and the reflection magnitude for the two ports obtained without any sample at the reference plane. $S_{11}$ and $S_{22}$ magnitudes are on the order of $-30 \mathrm{~dB}$, and the amplitude and phase of $S_{21}$ and $S_{12}$ are within $\pm 0.3 \mathrm{~dB}$ and $\pm 3^{\circ}$, respectively. We finalized the development of an experimentally confirmed homemade complex transmission-reflection setup by implementing a computer control code that measures and records the eight parameters sequentially.

\section{CHARACTERIZATION OF THE DESIGNED ABSORBERS}

\section{A. Type I absorber based on SRR}

In this case, we have the 2D SRR array placed in front of an aluminum back plate. In Fig. 4 we show the magnitude of the measured S-parameters. The separation between the back plate and the SRR layer was $d_{s}=7.2 \mathrm{~mm}$. At around the magnetic resonance frequency of the SRR, we find the expected reflection dip: $-12.8 \mathrm{~dB}$ at $4.74 \mathrm{GHz}$. As expected, the structure behaves as a resonant absorber. When the electromagnetic field impinges on the structure from port 1, the

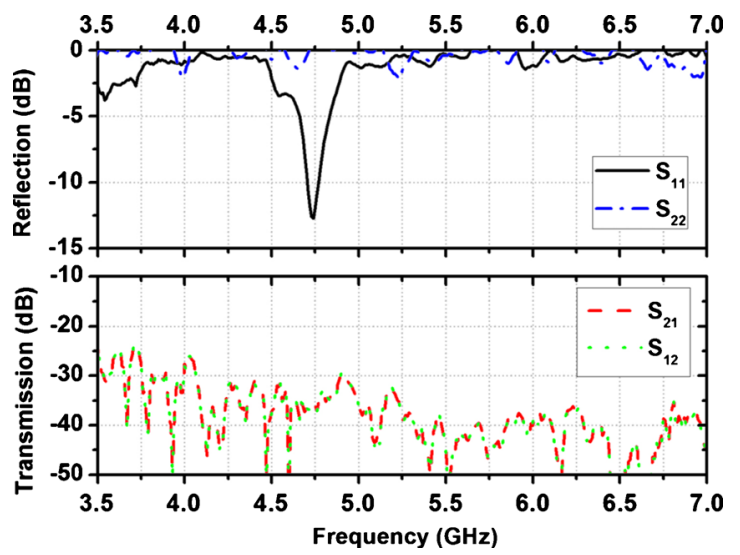

FIG. 4. (Color online) Scattering parameter amplitude for the type I absorber.

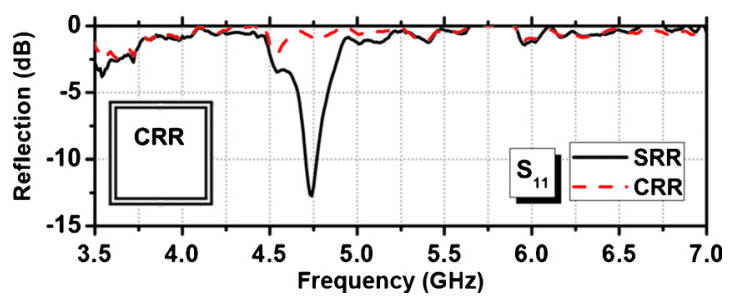

FIG. 5. (Color online) Comparison of the reflection responses (amplitude of $\mathrm{S}_{11}$ ) of the two absorbers made of SRR and CRR.

absorbance of the structure is calculated by the formula: $A$ $=1-\left|\mathrm{S}_{11}\right|^{2}-\left|\mathrm{S}_{21}\right|^{2}$. Our absorption peak at $4.74 \mathrm{GHz}$ was $94.7 \%$ and the $3 \mathrm{~dB}$ bandwidth is $\Delta f=370 \mathrm{MHz}$. The fractional bandwidth of the device is, thus $\Delta f / f_{0}=8 \%$. The minimum absorption value at the $3 \mathrm{~dB}$ bandwidth was $45.2 \%$. The total thickness of the absorber is $\tau=d+d s+p$ $=11.1 \mathrm{~mm}$, which corresponds to $\lambda / 5.7$ at the central frequency of operation.

In order to prove the magnetic origin of the resonant absorption, we applied a typical test for the analysis of the magnetic resonance in metamaterial structures. ${ }^{6,9,12,18,22}$ According to this technique, we closed the split of the SRR, considering the structure reported in the inset of Fig. 5 and referred to as a closed split-ring resonator (CRR). The electrical resonance response of the SRR does not change when we short the splits.

However, the only source of magnetic resonance of the SRR is the circulating currents that are driven by the split capacitance. As we short the split capacitance, the driven force of the circulating currents is eliminated and as a result, we expect to lose the absorbing peak at around the magnetic resonance frequency. We constructed a layer of a $2 \mathrm{D}$ CRR medium and measured its response in the absorber configuration. The results are reported in Fig. 5. The absence of the reflection minimum proves that the origin of the SRR based absorber was indeed the magnetic resonance of the SRR themselves.

While investigating the absorber performance, we show that the reflection minimum depends on the distance between the metamaterial layer and back plate. In Fig. 6, we compare the absorber performance for different $d_{s}$ values, ranging from 2.4 to $9.6 \mathrm{~mm}$. We observe that the electrical length of

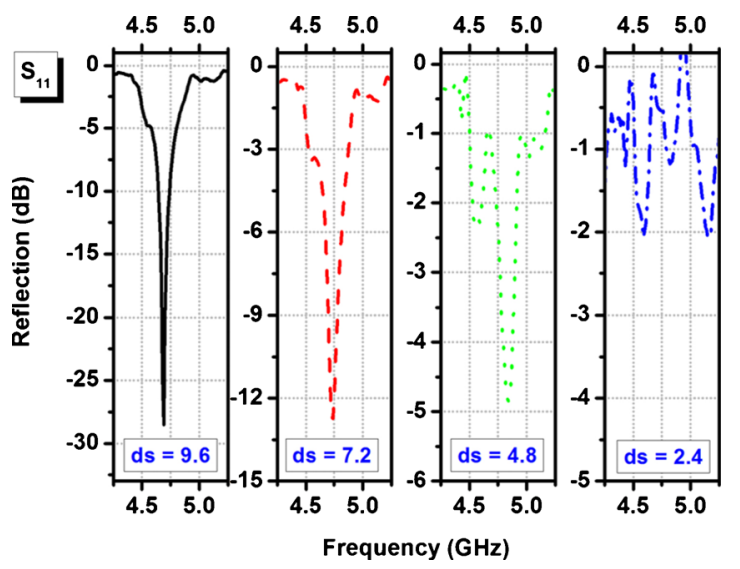

FIG. 6. (Color online) Dependence of the reflection minima on the separation between the metal plate and the metamaterial layer. 


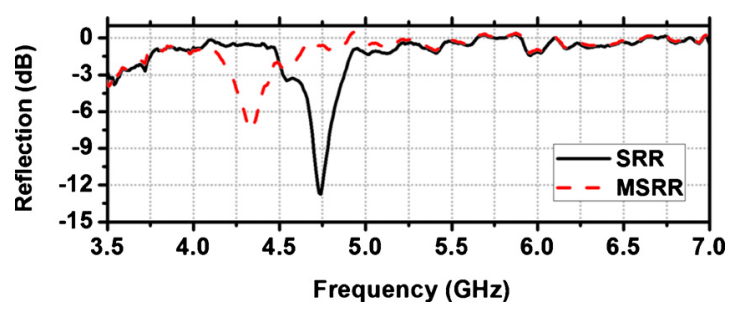

FIG. 7. (Color online) Effect of the resonator electrical size on the absorber thickness.

the separation between the metamaterial layer and the metal plate should be on the order of $\lambda / 8$ for high resonance strength. The absorbance increases for $d_{s}=9.6 \mathrm{~mm}$ and decreases when it is 4.8 or $2.4 \mathrm{~mm}$. At $d_{s}=9.6 \mathrm{~mm}$, the absorption peak is $99.8 \%$ at $4.69 \mathrm{GHz}$ with $\tau=\lambda / 4.7$ and FBW $=8 \%$. The minimum absorption value at the $3 \mathrm{~dB}$ bandwidth was $47.6 \%$. As $d_{s}$ further decreases, the response dies out. At this point we would like to quantitatively give the effect of substrate losses on the absorption values. We calculated absorption values numerically for by using lossy and lossless FR-4 substrates. FR-4 losses changes the absorption peak at a rate on the order of $0.01 \%$. Basically, the high amplitude currents flowing along the inclusions' metallic strips at resonance are much more responsible of the losses than what the dielectric material is. Inside the material, in fact, the magnetic field is high but the electric field is not as high as in Salisbury screens' operation. It might be possible that we are able to further reduce the absorber electrical thickness by using more miniaturized resonators, according to the methods that were developed in our recent papers. ${ }^{17,19,46}$

\section{B. Type I absorber based on MSRR}

In this part of the study, we show that by reducing the electrical size of the resonator, it is, indeed, possible to reduce the electrical thickness of the absorber. For the concept demonstration, we replaced the SRR medium with an MSRR one. The structural parameters of the MSRR are the same as the SRR but now the number of the rings is $N=8$ instead of $N=2$. This enabled us to obtain a smaller electrical size for the same space occupancy. Figure 7 shows that the reflection minimum shifts to lower frequencies in accordance with the resonator electrical size. In this configuration, the separation between the metamaterial layer and the metal plate is $d_{s}$ $=7.2 \mathrm{~mm}$. The absorption peak is $82 \%$ with $\mathrm{FBW}=6 \%$ and an electrical size of $\lambda / 6.2$ at $4.33 \mathrm{GHz}$. The minimum absorption value at the $3 \mathrm{~dB}$ bandwidth was calculated as $39.1 \%$. A further reduction in the absorber electrical dimensions can be easily obtained by using more miniaturized inclusions, such as the spiral resonators. ${ }^{17,19,27,46}$

In all the measurements presented so far, we have characterized the absorber for normal incidence only. Recently, we demonstrated that the response of SRR based metamaterials remains nearly the same up to $45^{\circ}$ incidence angle. ${ }^{47}$ Moreover, the metamaterial particles studied here can be packed into a 2D isotropic unit cell such that a polarization independent response can be obtained. ${ }^{17,48}$

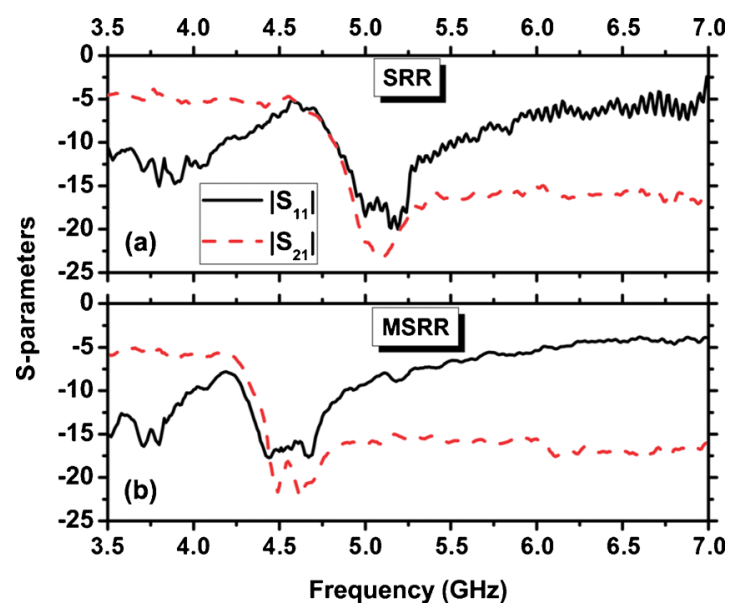

FIG. 8. (Color online) Scattering parameter amplitudes (decibel) for the type II absorber based on SRR and MSRR.

\section{Type II absorber based on SRR and MSRR}

In this part of the study, we replaced the metal plate with a carbon based resistive sheet having a sheet resistance of $5 \mathrm{k} \Omega$. In this configuration, the metamaterial layer is the back plate and the resistive sheet is the front layer. It is worth noticing that, in contrast to the type I absorber design, in this case we do not use any metal screen. Moreover, the resonance frequency depends on the magnetic inclusions (see type I). This kind of absorber is very useful when we would like to suppress a resonant peak of the radar signature of a nonmetallic object, made, for instance, of new ultralight materials based on carbon fibers. The absence of the metal backing, in this case, is crucial because if we used a metal backed absorber (e.g., a Salisbury screen), we would reduce the resonant peaks of the radar response at the desired frequency but we would increase the radar response at other frequencies. As presented in Ref. 49, in fact, the metal backing makes the nonmetallic object a metallic one.

The type II absorber should have real applications in navy ships to reduce the radar scattering cross section of some parts of the ship. These parts could be covered with type II absorbers in which flexible printed circuit board substrates could be utilized. The transmission and reflection data for this case are shown in Fig. 8. The configurations employing both the SRR and MSRR based media were measured. There is a $97.4 \%$ absorption peak at $4.67 \mathrm{GHz}$ for the MSRR case and a $98.4 \%$ absorption peak at $5.15 \mathrm{GHz}$ for the SRR case. The minimum absorption values at the $3 \mathrm{~dB}$ bandwidth were $46.5 \%$ and $46.9 \%$ for the MSRR and SRR cases, respectively. The separation between the metamaterial layer and the resistive sheet determines the frequency of the reflection dip: $d_{s} \approx 7 \mathrm{~mm}$ and $d_{s} \approx 10 \mathrm{~mm}$ have been used for the SRR and MSRR cases, respectively. Thereby, the electrical thicknesses and $-10 \mathrm{~dB}$ bandwidths $\left(\mathrm{BW}_{-10 \mathrm{~dB}}\right)$ are $\lambda / 4.7$, $\lambda / 4.2$ and $9.9 \%, 9.6 \%$, for the SRR and MSRR cases, respectively.

\section{DISCUSSION}

The main difference of type I absorber with Salisbury screen is that, conceptually, the resonance of the proposed 
design does not depend on the distance between the metallic plate and the absorbing layer. In fact, the frequency of operation is determined by the resonance of the magnetic inclusions used for the metamaterial absorbing layer. As we have clearly shown in our manuscript, more miniaturized inclusions (e.g., MSRR instead of SRR) lead to thinner absorbers. This concept can be scaled down by using ever more miniaturized inclusions. This approach is not viable in Salisbury screens. Another advantage of type I absorber over Salisbury screen is the angular bandwidth of operation. Typical values for Salisbury screens are around $30^{\circ}$, while, in our design, when the magnetic field is polarized along the axis of the magnetic inclusions, the angular bandwidth extends well beyond. Furthermore, we can obtain rather wide band high quality absorbers by merging a metamaterial type I absorber and Salisbury screen at any frequency band of the electromagnetic spectrum. This advantage is in parallel with the scalability of metamaterial based structures.

The metamaterial based absorber structures have some advantages over the ferromagnetic microwire based absorbers. ${ }^{41,42}$ The metamaterial absorbers are scalable to operate at the desired bands of the electromagnetic spectrum and their bandwidth can be designed to be narrow for specific applications. On the other hand, the ferromagnetic based absorbers can be tuned by external magnetic field and can provide electrically thin designs that can be fabricated by using special techniques. Another important advantage, which is common to the two types of absorbers proposed in the paper is the possibility to make them tunable, by tuning the response of the magnetic inclusions. As it has been widely demonstrated in the literature, in fact, the response of magnetic resonators can be easily tuned at microwave frequencies by using varactor loads ${ }^{50}$ or photoconductive switches. ${ }^{51}$

In this experimental study, we obtained absorbers with electrical sizes of $\lambda / 4.2, \lambda / 4.7$, and $\lambda / 5.7$. As we were limited with the low cost printed circuit board technology, we could demonstrate the proof of concept absorber thicknesses in the experiments. As previously explained, in fact, since the electrical thicknesses of the absorbers depend on the resonator electrical size the absorber thicknesses can have smaller electrical thicknesses. On the other hand, the cost of the printed circuit boards would be much higher for the fabrication of such resonator arrays. In this study, we tried to demonstrate the idea experimentally with an affordable cost.

For the type I absorber a real world application could be to cover a vehicle to reduce its radar cross section. For almost all vehicles metal back plate is already present. We can add a resonator layer on the top of an electromagnetically transparent spacer layer to cover the vehicles moving with moderate speed.

\section{CONCLUSION}

To sum up, the concept of metamaterials has proven to be useful in yet another field of microwave engineering, i.e., microwave absorbers. For a metal backed metamaterial absorber, we demonstrated the relation between the electrical thickness and the absorbance peak. The origin of the absor- bance was proven to be the magnetic resonance of the constituting artificial magnetic material inclusions. For approximately $\lambda / 5$ of total electrical thickness, we achieved an almost perfect absorption with a $8 \%$ fractional bandwidth by using SRR of $\lambda / 10$ electrical size. As we used metamaterial elements of a smaller electrical size, such as MSRR, we were able to reduce the absorber thickness accordingly. Moreover, we demonstrated another type of absorber: a metamaterial backed resistive sheet. Almost perfect absorbance was also achieved for this case, with $\lambda / 5$ total electrical thickness and $8 \%$ fractional bandwidth. These proofs of concepts may open the door to (a) even more miniaturized microwave absorbers, employing deeply subwavelength magnetic inclusions and (b) tunable devices employing either externally controlled capacitors connected to the magnetic resonators or lightinduced conductivity changes in the material filling the splits of the SRR and MSRR. These concepts can be also extended to the terahertz and infrared regimes of the electromagnetic spectrum, ${ }^{47}$ by scaling the physical size of the metamaterial elements and taking into account the material properties of metals, which, due to the increased losses, may, indeed, help to reduce the complexity of the designs.

\section{ACKNOWLEDGMENTS}

This work is supported by the European Union under the projects EU-PHOME, EU-ECONAM, and TUBITAK under Project Nos. 107A004 and 107A012. One of the authors (E.O.) also acknowledges partial support from the Turkish Academy of Sciences.

${ }^{1}$ W. W. Salisbury, U.S. Patent No. 2599944 (10 June 1952).

${ }^{2}$ R. A. Shelby, D. R. Smith, and S. Schultz, Science 292, 77 (2001).

${ }^{3}$ M. C. K. Wiltshire, J. B. Pendry, I. R. Young, D. J. Larkman, D. J. Gilderdale, and J. V. Hajnal, Science 291, 849 (2001).

${ }^{4}$ J. B. Pendry, D. Schurig, and D. R. Smith, Science 312, 1780 (2006).

${ }^{5}$ D. R. Smith, J. B. Pendry, and M. C. K. Wiltshire, Science 305, 788 (2004).

${ }^{6}$ Th. Koschny, M. Kafesaki, E. N. Economou, and C. M. Soukoulis, Phys. Rev. Lett. 93, 107402 (2004).

${ }^{7}$ S. Guenneau, S. A. Ramakrishna, S. Enoch, S. Chakrabarti, G. Tayeb, and B. Gralak, Photon. Nanostructures 5, 63 (2007).

${ }^{8}$ L. Zhang, G. Tuttle, and C. M. Soukoulis, Photonics Nanostruct. Fundam. Appl. 2, 155 (2004).

${ }^{9}$ K. B. Alici and E. Ozbay, Photonics Nanostruct. Fundam. Appl. 6, 102 (2008).

${ }^{10}$ N. Katsarakis, G. Konstantinidis, A. Kostopoulos, R. S. Penciu, T. F. Gundogdu, M. Kafesaki, E. N. Econoumou, T. Koschny, and C. M. Soukoulis, Opt. Lett. 30, 1348 (2005).

${ }^{11}$ K. Buell, H. Mosallaei, and K. Sarabandi, IEEE Trans. Microwave Theory Tech. 54, 135 (2006)

${ }^{12}$ E. Ozbay, K. Aydin, E. Cubukcu, and M. Bayindir, IEEE Trans. Antennas Propag. 51, 2592 (2003).

${ }^{13}$ K. Aydin, I. Bulu, and E. Ozbay, Appl. Phys. Lett. 90, 254102 (2007).

${ }^{14}$ C. M. Soukoulis, M. Kafesaki, and E. N. Economou, Adv. Mater. 18, 1941 (2006).

${ }^{15}$ K. B. Alici and E. Ozbay, J. Appl. Phys. 101, 083104 (2007).

${ }^{16}$ K. B. Alici and E. Özbay, Phys. Status Solidi B 244, 1192 (2007).

${ }^{17}$ K. B. Alici, F. Bilotti, L. Vegni, and E. Ozbay, Appl. Phys. Lett. 91, 071121 (2007).

${ }^{18}$ K. B. Alici and E. Ozbay, J. Phys. D: Appl. Phys. 41, 135011 (2008).

${ }^{19}$ K. B. Alici, F. Bilotti, L. Vegni, and E. Ozbay, Opt. Express 17, 5933 (2009).

${ }^{20}$ K. B. Alici and E. Ozbay, New J. Phys. 11, 043015 (2009).

${ }^{21}$ K. B. Alici and E. Ozbay, J. Opt. Soc. Am. B 26, 1688 (2009).

${ }^{22}$ K. B. Alici and E. Ozbay, IEEE J. Sel. Top. Quantum Electron. 16, 386 (2010). 
${ }^{23}$ A. Alu, F. Bilotti, N. Engheta, and L. Vegni, IEEE Trans. Antennas Propag. 55, 1698 (2007).

${ }^{24}$ F. Bilotti, A. Alu, and L. Vegni, IEEE Trans. Antennas Propag. 56, 1640 (2008).

${ }^{25}$ F. Bilotti, S. Tricarico, and L. Vegni, New J. Phys. 10, 115035 (2008).

${ }^{26}$ F. Bilotti, L. Scorrano, E. Ozbay, and L. Vegni, J. Opt. A: Pure Appl. Opt. 11, 114029 (2009)

${ }^{27}$ A. Alu, F. Bilotti, N. Engheta, and L. Vegni, IEEE Trans. Antennas Propag. 55, 13 (2007).

${ }^{28}$ C. G. Parazzoli, R. B. Greegor, K. Li, B. E. C. Koltenbah, and M. Tanielian, Phys. Rev. Lett. 90, 107401 (2003).

${ }^{29}$ J. B. Pendry, Nature Mater. 5, 599 (2006).

${ }^{30}$ A. Alu, F. Bilotti, N. Engheta, and L. Vegni, IEEE Trans. Antennas Propag. 55, 13 (2007).

${ }^{31}$ F. Qureshi, M. A. Antoniades, and G. V. Eleftheriades, IEEE Antennas Wirel. Propag. Lett. 4, 333 (2005).

${ }^{32}$ C. R. S. M. E. Ermutlu, M. K. Karkkainen, P. Ikonen, S. A. Tretyakov, and A. A. Sochava, International Workshop on Antenna Technology: Small Antennas and Novel Metamaterials (IEEE, New York, 2005), pp. 87-90. ${ }^{33}$ P. Ikonen, S. Maslovski, C. Simovski, and S. Tretyakov, IEEE Trans. Antennas Propag. 54, 1654 (2006).

${ }^{34}$ S. Hrabar, J. Bartolic, and Z. Sipus, IEEE Trans. Antennas Propag. 53, 110 (2005).

${ }^{35}$ N. Fang and X. Zhang, Appl. Phys. Lett. 82, 161 (2003).

${ }^{36}$ D. J. Kern and D. H. Werner, Microwave Opt. Technol. Lett. 38, 61 (2003).

${ }^{37}$ F. Bilotti, A. Alu, N. Engheta, and L. Vegni, Proceedings of the 2005 Nanoscience and Nanotechnology Symposium-NN2005, Frascati, Italy, 14-16 November 2005.
${ }^{38}$ H. Mosallaei and K. Sarabandi, Proceedings of 2005 IEEE Antennas and Propagation Society International Symposium, 2005, Vol. 1B, pp. 615618

${ }^{39}$ N. I. Landy, S. Sajuyigbe, J. J. Mock, D. R. Smith, and W. J. Padilla, Phys. Rev. Lett. 100, 207402 (2008).

${ }^{40}$ A. Labrador, C. Gómez-Polo, J. I. Pérez-Landazábal, V. Zablotskii, I. Ederra, R. Gonzalo, G. Badini-Confalonieri, and M. Vázquez, Opt. Lett. 35, 2161 (2010).

${ }^{41}$ P. Marin, D. Cotina, and A. Hernando, J. Magn. Magn. Mater. 290-291, 1597 (2005).

${ }^{42}$ P. Marin, D. Cortina, and A. Hernando, IEEE Trans. Magn. 44, 3934 (2008).

${ }^{43}$ F. Bilotti, L. Nucci, and L. Vegni, Microwave Opt. Technol. Lett. 48, 2171 (2006).

${ }^{44}$ J. Zhou, T. Koschny, M. Kafesaki, E. N. Economou, J. B. Pendry, and C. M. Soukoulis, Phys. Rev. Lett. 95, 223902 (2005).

${ }^{45}$ D. K. Ghodgaonkar, V. V. Varadan, and V. K. Varadan, IEEE Trans. Instrum. Meas. 38, 789 (1989).

${ }^{46}$ F. Bilotti, A. Toscano, L. Vegni, K. Aydin, K. B. Alici, and E. Ozbay, IEEE Trans. Microwave Theory Tech. 55, 2865 (2007)

${ }^{47}$ K. B. Alici and E. Ozbay, Opt. Lett. 34, 2294 (2009).

${ }^{48}$ J. B. Pendry, A. J. Holden, D. J. Robbins, and W. J. Stewart, IEEE Trans. Microwave Theory Tech. 47, 2075 (1999).

${ }^{49}$ F. Bilotti and L. Vegni, Metamaterials and Plasmonics: Fundamentals, Modeling, Applications (Springer, The Netherlands, 2009).

${ }^{50}$ I. Gil, J. Garcia-Garcia, J. Bonache, F. Martin, M. Sorolla, and R. Marques, Electron. Lett. 40, 1347 (2004).

${ }^{51}$ C. J. Panagamuwa, A. Chauraya, and J. C. Vardaxoglou, IEEE Trans. Antennas Propag. 54, 449 (2006). 